\title{
Insatisfação com a imagem corporal e fatores associados em mulheres do sul do Brasil
}

\author{
Dissatisfaction with self-image and its related factors in southern Brazilian women
}

\author{
Taiara Scopel Poltronieria, Cíntia Tusset ${ }^{b}$, Maria Luísa de Oliveira Gregolettoc, Cleber Cremonese ${ }^{d}$ \\ a Nutricionista graduada pela da Faculdade da Serra Gaúcha (FSG). \\ b Biomédica. Doutora em Ciências pelo Programa de Endocrinologia da Faculdade de Medicina da Universidade de São Paulo. \\ Pesquisadora da Unidade de Endocrinologia do Hospital de Clínicas de Porto Alegre. \\ c Nutricionista. Mestre em Saúde Coletiva pela Unisinos. Docente da FSG. \\ d Biólogo. Doutor em Ciência - Saúde Pública e Meio Ambiente pela Escola Nacional de Saúde Pública Sergio Arouca - ENSP/Fiocruz-RJ. Docente da FSG. \\ Fonte de financiamento: Bolsista PROBIC SPI no 0690-2551/14-4 - Fundação de Amparo à Pesquisa do Estado do Rio Grande do Sul (FAPERGS).
}

RESUMO

Objetivo: Associar a insatisfação com a imagem corporal (IC) com fatores sócio demográficos, estado nutricional e transtornos alimentares em mulheres adultas do município de Ipê/RS.

Materiais e Métodos: Estudo transversal, realizado com 187 mulheres entre 18 e 59 anos. Aplicou-se um questionário padronizado, composto por informações sócio demográficas, avaliação da insatisfação com a IC (Body Shape Questionnaire) e de sintomas para transtornos alimentares (Questionário EAT-26). Além disso, foram aferidos peso e estatura para o cálculo do Índice de Massa Corporal (IMC). Na análise estatística, frequências foram realizadas, além de teste de tendência linear, qui-quadrado e regressão de Poisson para identificar associações entre o desfecho e fatores de exposição. Foi adotando um nível de significância de 5\%.

Resultados: Do total, 45,9\% das mulheres apresentaram insatisfação com a IC, 40\% estavam com excesso de peso, e 17,1\% apresentaram sintomas para transtorno alimentar. Estado nutricional e presença de sintomas para transtorno alimentar mostraram-se associadas com insatisfação da IC. Obesas apresentaram 49\% maior probabilidade de apresentar o desfecho em relação às eutróficas. Em relação aos sintomas para transtorno alimentar, mulheres com rastreamento positivo mostraram $61 \%$ maior probabilidade de ter insatisfação com seu corpo em comparação às mulheres que apresentaram rastreamento negativo para esta variável. Não foram encontradas associações estatisticamente significativas entre variáveis sócio demográficas insatisfação com a IC.

Conclusão: Os resultados do estudo sugerem uma elevada prevalência de insatisfação com a IC, independente da faixa etária e do local de moradia. Mulheres com excesso de peso e com sintomas de transtorno alimentar têm maior probabilidade de ter algum grau de insatisfação com a IC.

Palavras-chave: imagem corporal; transtornos da alimentação; estado nutricional; nutrição; saúde da mulher.

Objective: To associate the dissatisfaction with body self-image (BSI) with socio demographic factors, nutritional status and eating disorders in adult women of the municipality of Ipê/RS.

Material and Methods: A cross-sectional study was carried out with 187 women between the age of 18 and 59. To collect data, a standardized and validated questionnaire containing socio demographic information, assessment of dissatisfaction with BSI (Body Shape Questionnaire) and symptoms of eating disorders (Questionnaire EAT-26) was applied. In addition, all participants were measured by weight and height to calculate their Body Mass Index (BMI). In the statistical analysis, simple frequencies were used, in addition to linear trend test, chi-square and Poisson regression in order to identify associations between outcomes and exposure factors. A significance level of $5 \%$ was established.

Results: $45.9 \%$ of the women demonstrated dissatisfaction with their BSI, $40 \%$ were overweight, and $17.1 \%$ shown symptoms of eating disorders. Nutritional status and the presence of eating disorder symptoms were significantly associated with dissatisfaction of BSI. Obese women were $49 \%$ more likely to present symptoms of dissatisfaction in relation to eutrophic women. Regarding the variable used for eating disorder symptoms, women with positive screening showed $61 \%$ more probability to be dissatisfied with their body compared to women who had negative screening for this variable. There were no statistically significant associations between socio demographic variables and dissatisfaction with BSI.

Conclusion: These results suggest a high prevalence of dissatisfaction with BSI, regardless of age and place of residence. Women with excess weight and eating disorder symptoms are more likely to have some degree of BSI dissatisfaction.

Keywords: body image; eating disorders; nutritional status; nutrition; women's health.

\section{Correspondência:}

Maria Luísa de Oliveira GreGoletto

Faculdade da Serra Gaúcha - Curso de Nutrição

Os Dezoito do Forte, 2366

95020-472 Caxias do Sul, RS, Brasil

E-mail:mluisagrego@gmail.com 


\section{INTRODUÇÃO}

A imagem corporal (IC) pode ser definida como representações mentais acerca do tamanho, da aparência e da forma do corpo. A maneira com que cada indivíduo percebe o seu próprio corpo é influenciada tanto por imagens concebidas pela sua mente, como por seus pensamentos, sentimentos e suas experiências de $v^{1} \mathbf{d a}^{1,2}$. Nas últimas décadas, têm se vivenciado uma mudança em relação aos padrões de beleza estabelecidos pela sociedade ${ }^{3}$. Desse modo, observa-se um aumento expressivo da preocupação com a IC, especialmente na busca por um padrão de beleza idealizado ${ }^{4}$.

A insatisfação com a IC atinge principalmente as mulheres, devido a sua prevalência se mostrar superior em comparação à identificada entre os homens ${ }^{5,6}$. Entre os motivos que explicam o elevado grau de insatisfação com a IC na população feminina está a elevada cobrança social e a forte influência da mídia pela busca do corpo perfeito ${ }^{7,8}$. No Brasil, evidências apontam uma elevada prevalência de insatisfação com IC entre as mulheres adultas ${ }^{9,10}$.

Entre os desfechos desfavoráveis à saúde que têm sido demonstrados em mulheres insatisfeitas com a IC, está o aumento de risco para desenvolvimento de transtornos alimentares ${ }^{11,12}$. Além disso, a obesidade é considerada um mediador da insatisfação com a IC. Estudos apontam que indivíduos do sexo feminino com excesso de peso apresentam maior probabilidade de insatisfação com a IC quando comparados aos eutróficos ${ }^{6,13-16}$. Os transtornos alimentares e a obesidade, por sua vez, são considerados problemas de grande relevância para a saúde pública, em virtude da sua elevada prevalência e suas possíveis consequências físicas e psicossociais ${ }^{17}$.

Nesta perspectiva, o objetivo do presente estudo foi associar a insatisfação com a IC com fatores sócio demográficos, estado nutricional e transtornos alimentares em mulheres adultas do município de Ipê/RS.

\section{MATERIAIS E MÉTODOS}

Foi realizado um estudo com delineamento transversal observacional, o qual buscou identificar possíveis associações entre as características sócio demográficas (idade e local de moradia), de estado nutricional e de sintomas de transtornos alimentares em relação à insatisfação com a IC.

A população do estudo foi composta por mulheres adultas do município de Ipê, cidade localizada na região noroeste do estado do Rio Grande do Sul com população estimada em 6.347 habitantes, apresentando 2.933 mulheres $^{18}$. Foram incluídas mulheres com idade entre 18 a 59 anos de idade, moradoras da área urbana e rural do município.
O tamanho da amostra considerou a prevalência de insatisfação com a IC de $45 \%{ }^{9}$, com um erro máximo de $7 \%$ e nível de confiança de $95 \%$, obtendo-se um tamanho amostral de 183 mulheres.

A seleção das mulheres moradoras de domicílios urbanos ocorreu de forma aleatória sistemática, respeitando a proporção populacional dos setores censitários do município. Em cada setor sorteou-se a esquina de ponto de partida, em seguida foi realizado um novo sorteio para determinação do domicílio inicial entre as 5 primeiras casas, seguindo a contagem de forma sistemática e numa direção pré-estabelecida para percurso da quadra, com pulo de 5 casas, sempre incluindo na amostra o 6o domicílio. Já a seleção das mulheres moradoras de domicílios rurais ocorreu através de sorteio simples dos domicílios através do talão do agricultor, cadastrados na secretaria municipal da agricultura do município de Ipê.

Como critérios de inclusão foram selecionados aqueles domicílios sorteados, os quais apresentavam ao menos uma moradora do sexo feminino, com idade entre 18 e 59 anos, em condições físicas e mentais para responder ao questionário e para realização das aferições.

A coleta de dados ocorreu por meio de visitas domiciliares entre julho a dezembro de 2014, utilizando-se um questionário estruturado composto por informações sócio demográficas (idade coletada em anos completos - posteriormente categorizada em 18 a 29 anos, 30 a 39 anos, 40 a 49 anos e 50 a 59 anos; local de moradia - rural e urbano), de comportamentos de risco para transtornos alimentares (Questionário EAT-26 - Teste de Atitudes Alimentares) ${ }^{19}$, de insatisfação com a IC (Body Shape Questionnaire - Questionário Sobre o Corpo ${ }^{20}$ e de estado nutricional.

O estado nutricional foi avaliado pelo IMC, resultado da razão entre a massa corporal (em quilogramas) e o quadrado da estatura (em metros). Para aferição do peso das participantes, utilizou-se balança digital (modelo MEA 07700 Plenna $^{\circledR}$, São Paulo, Brasil), com precisão de $100 \mathrm{~g}$ e capacidade de $150 \mathrm{~kg}$, estando as mulheres sem sapatos e vestindo roupas leves. A estatura foi obtida por meio de estadiômetro portátil da marca Sanny ${ }^{\circledR}$, modelo carpice, com capacidade de $210 \mathrm{~cm}$ e precisão de $0,1 \mathrm{~cm}$. Ambas as medidas foram feitas em duplicatas e calculada a sua média. Foram consideradas eutróficas aquelas mulheres com IMC entre 18,5 e 24,9 kg/ $\mathrm{m}^{2}$, sobrepeso as participantes apresentando IMC entre 25 e $29,9 \mathrm{~kg} / \mathrm{m}^{2}$ e as que possuíam $I M C \geq 30 \mathrm{~kg} / \mathrm{m}^{2}$ foram consideradas obesas ${ }^{21}$.

Para verificação de presença de sintomas de anorexia e bulimia aplicou-se o Teste de Atitudes Alimentares (EAT-26), proposto por Garner et al. ${ }^{22}$, traduzido para o português 
por Nunes et al. ${ }^{23}$ e validado por Bighetti ${ }^{19}$. Este é um instrumento autoaplicável, sendo composto por questões que envolvem os sintomas de restrição alimentar e identifica comportamentos anoréxicos e de dieta. Cada questão possui seis opções de resposta, que pontuam entre 0 e 3 . Ao final, a pontuação deste instrumento questionário varia de 0 a 78 pontos $^{19,22}$. No presente estudo, mulheres que obtiveram pontuação igual ou maior a 21 pontos foram consideradas com rastreamento positivo para transtornos alimentares ${ }^{23}$.

Informações referentes ao desfecho foram coletadas através do questionário padronizado e validado BSQ (Body Shape Questionnaire - Questionário Sobre o Corpo), o qual avalia a insatisfação com a IC, validada em sua versão para o português ${ }^{20} \mathrm{e}$ elaborado originalmente por Cooper et al. ${ }^{24}$. O BSQ é um instrumento de autorrelato, que mensura o grau de insatisfação com a forma do corpo, composto por 34 questões, contendo seis opções de resposta, que pontuam de 1 a $6^{25}$. A classificação do BSQ foi realizada a partir da pontuação obtida pelas mulheres, refletindo o nível de preocupação com a IC: escores menores ou igual a 80 pontos foram considerados como um padrão de normalidade, traduzindo a ausência de insatisfação com a IC; escores entre 81 e 110 pontos, classificados como grau leve de insatisfação com a IC; escores entre 111 e 140 classificados como grau moderado de insatisfação com a IC e, por fim, escores acima de 140 pontos, classificados como presença de grave grau de insatisfação com a $\mathrm{IC}^{25}$.

Vale ressaltar que estes são instrumentos de autorrelato, e são utilizados em clínicas médicas para delimitar situações de síndromes precursoras de comportamento alimentar e de outras comorbidades psiquiátricas, contudo, as mulheres abordadas pelo presente estudo não foram submetidas à entrevista com fins de diagnóstico clínico ${ }^{26}$.

As análises estatísticas foram realizadas através do software Statistical Package for the Social Sciences da IBM Corporation (IBM SPSS ${ }^{\circledR)}$, versão 20.0 (IBM Corp. Armonk, Estados Unidos). Primeiramente, as variáveis categóricas do inquérito foram descritas através dos valores brutos e em frequências. Variáveis contínuas foram analisadas através de médias com seu respectivo desvio-padrão. Para avaliar a associação entre a insatisfação com a IC com variáveis de exposição, análises bivariadas foram realizadas através do teste de qui-quadrado e de tendência linear, ficando estabelecido um nível de significância estatística $p<0,05$. Na análise ajustada, calculou-se a razão de prevalência e regressão de Poisson, na qual se adotou como critério de inclusão, no modelo para a análise final, apenas aquelas variáveis que apresentaram um valor de $\mathrm{p}<0,20$ na análise bruta.

A pesquisa foi aprovada pelo Comitê de Ética em Pesquisa do Círculo Operário Caxiense - Faculdade da Serra
Gaúcha, obedecendo à Resolução no 466/12 do Conselho Nacional de Saúde, sob parecer no 671.692 e CAAE: 30457314.4.0000.5311. Todas as participantes do estudo foram informadas da natureza do trabalho, e assinaram o termo de consentimento livre e esclarecido.

\section{RESULTADOS}

Foram avaliadas 187 mulheres adultas da cidade de Ipê/ RS com média de idade foi $37 \pm 12$ anos, sendo que $58,3 \%$ das mulheres entrevistadas presentavam idade entre 18 e 39 anos. Em relação ao local de moradia, 75,9\% residiam em local urbano (Tabela 1).

Tabela 1. Características sociodemográficas, estado nutricional e variáveis comportamentais em mulheres adultas no município de Ipê-RS (2014).

\begin{tabular}{lcc}
\multicolumn{1}{c}{ Variáveis Categóricas } & n & $\%$ \\
Idade & & \\
$\quad 18$ a 29 anos & 62 & 33,2 \\
30 a 39 anos & 47 & 25,1 \\
40 a 49 anos & 41 & 21,9 \\
50 a 59 anos & 37 & 19,8 \\
Local de moradia & & \\
$\quad$ Urbano & 142 & 75,9 \\
$\quad$ Rural & 45 & 24,1 \\
Estado nutricional & & \\
$\quad$ Eutrofia & 111 & 59,4 \\
Sobrepeso & 57 & 30,5 \\
Obesidade & 19 & 10,2 \\
Presença de transtorno alimentar & & \\
$\quad$ Negativo & 155 & 82,9 \\
$\quad$ Positivo & 32 & 17,1 \\
Nível de insatisfação com a imagem corporal* & & \\
$\quad$ Ausente & 101 & 54,0 \\
Leve & 56 & 29,9 \\
Moderado & 21 & 11,2 \\
Grave & 9 & 4,8 \\
\hline
\end{tabular}

* Variável desfecho; DP: desvio padrão.

Quanto ao estado nutricional, notou-se que o peso e a estatura foram em média $66 \pm 10 \mathrm{~kg}$ e 1,63 $\pm 0,6 \mathrm{~m}$, com um IMC de $24,7 \pm 3,9 \mathrm{~kg} / \mathrm{m}^{2}$. Aproximadamente $40 \%$ das mulheres avaliadas estavam com excesso de peso. Em relação à presença de sintomas de transtornos alimentares, a prevalência encontrada no estudo foi de $17,1 \%$. Ao que se refere ao nível de insatisfação com a IC, destaca-se que $46 \%$ das entrevistadas apresentaram algum nível da presença do desfecho (Tabela 1).

$\mathrm{Na}$ análise bruta, a relação do desfecho "grau de insatisfação com a IC" com a idade e o local de moradia, não 
Tabela 2. Análise bruta entre o grau de insatisfação com a imagem corporal, variáveis sócio demográficas, estado nutricional e variáveis comportamentais de mulheres adultas no município de Ipê-RS (2014).

\begin{tabular}{|c|c|c|c|c|c|}
\hline \multirow{2}{*}{ Variáveis } & \multicolumn{4}{|c|}{ n (\%) } & \multirow{2}{*}{ Valor de $p$} \\
\hline & Ausente & Leve & Moderado & Grave & \\
\hline Idade & & & & & $0,429 * *$ \\
\hline 18 a 29 anos & $33(32,7)$ & $20(35,7)$ & $8(38,1)$ & $1(11,1)$ & \\
\hline 30 a 39 anos & $21(20,8)$ & $15(26,8)$ & $7(33,3)$ & $4(44,4)$ & \\
\hline 40 a 49 anos & $23(22,8)$ & $14(25,0)$ & $3(14,3)$ & $1(11,1)$ & \\
\hline 50 a 59 anos & $24(23,8)$ & $7(12,5)$ & $3(14,3)$ & $3(33,3)$ & \\
\hline Local de moradia & & & & & $0,353^{* *}$ \\
\hline Urbano & $75(74,3)$ & $43(76,8)$ & $15(71,4)$ & $9(100)$ & \\
\hline Rural & $26(25,7)$ & $13(23,2)$ & $6(28,6)$ & $0(0)$ & \\
\hline Estado nutricional & & & & & $0,004^{*}$ \\
\hline Eutrofia & $70(69,3)$ & $28(50,0)$ & $12(57,1)$ & $1(11,1)$ & \\
\hline Sobrepeso & $26(25,7)$ & $21(37,5)$ & $5(23,8)$ & $5(55,6)$ & \\
\hline Obesidade & $5(5,0)$ & $7(12,5)$ & $4(19,0)$ & $3(33,3)$ & \\
\hline Presença de Transtorno Alimentar & & & & & $<0,001^{* *}$ \\
\hline Negativo & $95(94,1)$ & $44(78,6)$ & $14(66,7)$ & $2(22,2)$ & \\
\hline Positivo & $6(5,9)$ & $12(21,4)$ & $7(33,3)$ & $7(77,8)$ & \\
\hline
\end{tabular}

*Teste de tendência linear; ${ }^{* *}$ Teste qui-quadrado.

apresentou associação significativa. No entanto, observou-se, em relação ao estado nutricional, que quanto maior o IMC, maior também o grau de insatisfação com a IC $(p=0,004)$. Ainda, mulheres que apresentaram sintomas para transtorno alimentar, apresentaram maiores prevalências do desfecho $(p<0,001)$ (Tabela 2).

Os resultados da análise ajustada sugerem que ambas as variáveis, estado nutricional e sintomas de transtorno alimentar, estão associadas com a insatisfação com a IC $(p<0,001)$. Mulheres com obesidade apresentaram $49 \%$ maior probabilidade de apresentar o desfecho em relação às mulheres eutróficas. Já em relação aos sintomas de transtorno alimentar, mulheres com essa característica apresentaram $61 \%$ maior probabilidade de ter insatisfação com a IC quando comparadas às mulheres sem sintomas para esse transtorno (Tabela 3).

Tabela 3. Análise ajustada entre a insatisfação com a imagem corporal, estado nutricional e variável comportamental de mulheres adultas no município de Ipê-RS (2014).

\begin{tabular}{lcc}
\multicolumn{1}{c}{ Variáveis } & RP (IC 95\%) & Valor de p \\
Estado nutricional & & $<0,001$ \\
$\quad$ Eutrofia & 1 & \\
Sobrepeso & $1,12(0,97-1,29)$ & \\
Obesidade & $1,49(1,21-1,82)$ & \\
Presença de transtorno Alimentar & & $<0,001$ \\
$\quad$ Negativo & 1 & \\
Positivo & $1,61(1,38-1,88)$ & \\
\hline
\end{tabular}

* Análise multivariada por meio de regressão de Poisson.

\section{DISCUSSÃO}

O presente estudo avaliou a frequência da insatisfação com a IC e fatores sócio demográficos, de local de moradia, de estado nutricional e de sintomas de transtornos alimentares em mulheres adultas de um município do interior do Rio Grande do Sul. A identificação de uma maior frequência de insatisfação com a IC entre indivíduos do sexo feminino é bem esclarecida pela literatura ${ }^{27,28}$. Este fato possivelmente ocorre devido à excessiva cobrança pela sociedade e pela mídia de um corpo magro, principalmente entre adolescentes e mulheres adultas jovens ${ }^{29}$. Os autores identificaram que grande parte dos estudos com indivíduos saudáveis investigaram adolescentes do sexo feminino e em mulheres adultas jovens ${ }^{30-34}$. Entretanto, revisão sistemática recente apontou a preocupação com o corpo, assim como as suas consequências, como um aspecto significativo entre mulheres de meia-idade ${ }^{35}$.

Nesta perspectiva, a escolha de investigar mulheres adultas justifica-se pelo fato da maior frequência de insatisfação com a IC no sexo feminino quando comparado aos homens ${ }^{27,28,36,37}$. Além disso, poucos estudos em mulheres adultas investigando insatisfação com a IC foram encontrados. Evidências relevam que as mulheres encontram-se mais suscetíveis aos parâmetros de beleza impostos pela sociedade moderna e por outras situações psicológicas ${ }^{38}$, que tornam o estereotipo caracterizado como ideal, um objeto de fortalecimento pessoal e profissional ${ }^{29}$. 
Os resultados desta investigação demonstraram que, independentemente da faixa etária, quase metade $(45,9 \%)$ das entrevistadas estavam insatisfeitas com o seu corpo. Pesquisas internacionais apontaram prevalência de insatisfação com o seu corpo superior ao do presente estudo. Estudo conduzido na Alemanha identificou, entre as 2.123 mulheres investigadas, uma prevalência de 48,3\% de insatisfação com o seu corpo ${ }^{28}$. Níveis de IC superiores foram observados em dois estudos realizados nos Estados Unidos, onde $73 \%$ e $71 \%$, respectivamente, das mulheres de meia-idade relataram insatisfação com o seu peso ${ }^{39,40}$.

No Brasil, estudo conduzido no município de Florianópolis/SC mostrou uma prevalência de insatisfação com a IC entre as universitárias de uma instituição pública de $47,3 \%{ }^{9}$, resultado esse semelhante ao encontrado no presente estudo. Frequência superior $(57,7 \%)$ foi identificada entre mulheres de 12 a 39 anos praticantes de atividade física de uma academia, no interior do estado de São Paulo $^{10}$. Em contrapartida, alguns dados demonstraram uma prevalência inferior de insatisfação com a IC em comparação à demonstrada nesta investigação. Resultados referentes a universitárias de Ouro Preto/MG e do Rio de Janeiro/RJ apontaram uma prevalência de insatisfação com a IC de $13,7 \%$ e $27,7 \%$, respectivamente ${ }^{12,33}$.

Aponta-se que a elevada prevalência de insatisfação com a IC identificada nos estudos pode estar diretamente associada ao contexto social, na qual a mídia exerce forte influência ${ }^{7,8}$. Embora este não seja o único fator a ser considerado, observa-se que principalmente a televisão e a internet têm contribuído na divulgação do corpo perfeito, tendo grande peso sobre a valorização do culto aos músculos e a conquista de corpos cada vez mais magros e rejuvenescidos ${ }^{41,42}$.

Os sintomas de transtornos alimentares e o excesso de peso demonstraram, neste estudo, associação com a insatisfação com a IC. Estas associações também foram identificadas por outros autores ${ }^{10,12,32,36,37}$. A relação entre presença de transtornos alimentares com a insatisfação com a IC foi identificada em um estudo conduzido na Espanha com 680 indivíduos de ambos os $\operatorname{sexos}^{43}$. Outro estudo internacional com mulheres australianas, entre 18 e 55 anos de idade, observou mais insatisfação com a IC entre aquelas com transtornos alimentares em comparação com as que não apresentaram nenhum transtorno ${ }^{44}$.

No Brasil, encontrou-se associação entre a insatisfação com a IC e o agravamento dos sintomas comportamentais alimentares em mulheres praticantes de atividade física de uma academia. Resultados semelhantes foram observados entre universitárias frequentadoras de academias de duas universidades, onde a insatisfação moderada e grave da IC estava associada ao comportamento alimentar de risco ${ }^{10,32}$.
Estes achados devem ser vistos como uma forma de alerta a respeito das consequências geradas pela supervalorização de um corpo idealizado, sobretudo o surgimento de transtornos alimentares. Desta forma, o estímulo à discussão a respeito da aceitação do seu corpo, assim como, a prevenção e detecção dos fatores relacionados ao desenvolvimento dos transtornos alimentares se mostram primordiais $\mathrm{s}^{9,41}$.

A associação entre a insatisfação com a IC e estado nutricional foi observada em investigação realizada na Alemanha por mulheres com sobrepeso, as quais apresentaram maiores chances de estar insatisfeitas com o seu corpo ${ }^{28}$. Já, um estudo longitudinal conduzido no Canadá, num período de 10 anos, demonstrou associação entre insatisfação com a IC e IMC nas participantes do sexo feminino. Ao longo desse período tanto os escores de insatisfação com a IC, quantos os de IMC tiveram tendência linear ${ }^{6}$. Corroborando com os resultados anteriores, Siervo et al. ${ }^{36}$ observaram entre mulheres italianas adultas-jovens uma prevalência menor de insatisfação com a IC entre as não obesas quando comparadas às mulheres com excesso de peso.

No Brasil, dados recentes da coorte de Pelotas/RS de 1982 revelaram que entre as mulheres as quais apresentaram aumento do IMC durante o período do estudo eram menos propensas a se percebem mais magras do que o ideal ${ }^{45}$. Em Ouro Preto/MG, estudo observou que entre as alunas do curso de nutrição com insatisfação da IC moderada e grave as médias de IMC foram superiores quando comparadas as que não apresentaram insatisfação com a $\mathrm{IC}^{12}$. Ainda, estudo conduzido na cidade de Uberlândia/MG corrobora com os presentes achados, que avaliaram mulheres entre 20 e 30 anos de idade, no qual a insatisfação com a IC ocorreu em $60 \%$ das mulheres eutróficas, $90 \%$ das com sobrepeso e $100 \%$ das obesas ${ }^{37}$.

Embora a identificação de elevados índices de insatisfação com a IC seja preocupante tanto em indivíduos com excesso de peso como em indivíduos eutróficos ${ }^{34}$, o primeiro grupo requer um olhar diferenciado. Pesquisadores têm demonstrado maior número de tentativas ineficazes de perder peso em indivíduos com sobrepeso e obesidade, o que ocasiona uma maior insatisfação com o seu corpo ${ }^{46,47}$. Outro ponto alarmante da presença de insatisfação com a IC em indivíduos com excesso de peso, é que estes estão mais propensos a desenvolver uma obsessão pela aparência física, levando ao comer desordenado ${ }^{48}$.

O presente estudo apresenta algumas limitações metodológicas. A ampliação do número de entrevistadas poderia identificar outros fatores associados que não foram evidenciados aqui. Outro aspecto a ser discutido, trata-se da complexidade da cadeia causal que determinam a presença 
de insatisfação com a IC, portanto sugere-se que a inclusão de outras variáveis para análise. Além disso, o delineamento transversal observacional também consiste num limitante. Desse modo, faz-se necessário um cuidado na interpretação dos dados apresentados diz respeito à possibilidade de causalidade reversa. Por outro lado, acredita-se que esse delineamento contemplou os objetivos propostos pelo estudo, ao estimar a frequência da presença da insatisfação com a IC e associação com fatores sócio demográficos, estado nutricional e presença de transtornos alimentares em mulheres residentes num município do interior do Rio Grande do Sul.

Os resultados obtidos pelo presente estudo sugerem elevada prevalência de insatisfação com a IC entre as mulheres investigadas, independente da faixa etária considerada e do local de moradia. Observou-se ainda, que a insatisfação com a IC foi associada à presença de sintomas de transtornos alimentares e ao excesso de peso. Sabe-se que a construção de uma IC negativa pode ocorrer ainda na infância e na adolescência, com tendência a se manter na vida adulta. A identificação precoce de insatisfação com a IC pode prevenir futuros problemas relacionados ao desencadeamento de transtornos alimentares. Nesta perspectiva, os dados do presente estudo podem contribuir para a implantação de estratégias e ações em saúde voltadas a melhor aceitação do corpo, assim como as consequências geradas pela insatisfação com a IC, no município de Ipê/RS.

\section{REFERÊNCIAS}

1. Almeida GAN, Santos JE, Pasian SR, Loureiro SR. Percepção de tamanho e forma corporal de mulheres: estudo exploratório. Psicol Estud. 2005;10(1):27-35. http://dx.doi.org/10.1590/S141373722005000100005

2. Slade PD. What is body image? Behav Res Ther. 1994;32(5):497502. http://dx.doi.org/10.1016/0005-7967(94)90136-8

3. Alvarenga MS, Dunker KLL, Philippi ST, Scagliusi FB. Influência da mídia em universitárias brasileiras de diferentes regiões. J Bras Psiquiatr. 2010;59(2):111-8. http://dx.doi.org/10.1590/S004720852010000200006

4. Santos ARM, Silva EAPC, Moura PV, Dabbico P, Silva PPC, Freitas CMSM. A busca pela beleza corporal na feminilidade e masculinidade. Rev Bras Ci Mov. 2013;21(2):135-42. http://dx.doi. org/10.18511/0103-1716/rbcm.v21n2p135-142

5. Freitas CMSM, Lima RBT, Costa AS, Lucena Filho A. O padrão de beleza corporal sobre o corpo feminino mediante o IMC. Rev Bras Educ Fís Esporte. 2010;24(3):389-404. http://dx.doi.org/10.1590/ S1807-55092010000300010

6. Bucchianeri MM, Arikian AJ, Hannan PJ, Eisenberg ME, NeumarkSztainer D. Body dissatisfaction from adolescence to young adulthood: findings from a 10-year longitudinal study. Body Image. 2013;10(1):1-7. http://dx.doi.org/10.1016/j.bodyim.2012.09.001
7. Fernandez S, Pritchard M. Relationships between self-esteem, media influence and drive for thinness. Eat Behav. 2012;13(4): 321-5. http://dx.doi.org/10.1016/j.eatbeh.2012.05.004

8. Alves TCHS, Santana MLP, Silva RCR, Pinto EJ, Assis AMO. Fatores associados a sintomas de transtornos alimentares entre escolares da rede pública da cidade do Salvador, Bahia. J Bras Psiquiat. 2012; 61(2):55-63. http://dx.doi.org/10.1590/S004720852012000200001

9. Costa LCF, Vasconcelos FAG. Influência de fatores socioeconômicos, comportamentais e nutricionais na insatisfação com a imagem corporal de universitárias em Florianópolis, SC. Rev Bras Epidemiol. 2010;13(4):665-76. http://dx.doi.org/10.1590/S1415790X2010000400011

10. Souza MCDFP, Souza LV, Barroso SM, Scorsolini-Comin F. Padrões alimentares e imagem corporal em mulheres frequentadoras de academia de atividade física. Psico-USF. 2013;18(3):445-54. http:// dx.doi.org/10.1590/S1413-82712013000300011

11. Guimarães AD, Machado SP, França AKTC, Calado IL. Transtornos alimentares e insatisfação com a imagem corporal em bailarinos. Rev Bras Med Esporte. 2014;20(4):267-71. http://dx.doi. org/10.1590/1517-86922014200401399

12. Silva JD, Silva ABJ, Oliveira AVK, Nemer ASA. Influência do estado nutricional no risco para transtornos alimentares em estudantes de nutrição. Ciên Saúde Coletiva. 2012;17(12):3399-406. http:// dx.doi.org/10.1590/S1413-81232012001200024

13. Miranda VPN, Filgueiras JF, Neves CM, Teixeira PC, Ferreira MEC. Insatisfação corporal em universitários de diferentes áreas de conhecimento. J Bras Psiquiatr. 2012;61(1):25-32. http://dx.doi. org/10.1590/S0047-20852012000100006

14. Alvarenga MS, Philippi ST, Lourenço BH, Sato PM, Scagliusi FB. Insatisfação com a imagem corporal em universitárias brasileiras. J Bras Psiquiatr. 2010;59(1):44-51. http://dx.doi.org/10.1590/S004720852010000100007

15. Bosi MLM, Luiz RR, Morgado CMC, Costa MLS, Carvalho RJ. Autopercepção da imagem corporal entre estudantes de nutrição: um estudo no município do Rio de Janeiro. J Bras Psiquiatr. 2006;55(2):108-13. http://dx.doi.org/10.1590/S004720852006000200003

16. Quadros TMB, Gordia AP, Martins CR, Silva DAS, Ferrari EP, Petroski EL. Body image among university students: association with nutritional status and gender. Motriz Rev Edu Fis. 2010;16(1):78-85.

17. Macpherson-Sanchez AE. Integrating fundamental concepts of obesity and eating disorders: implications for the obesity epidemic. Am J Public Health. 2015;105(4):e71-85. http://dx.doi.org/10.2105/ AJPH.2014.302507

18. IBGE. Estimativas populacionais para os municípios brasileiros em 01.07.2012 [Internet]. 2012 [cited 2014]. Available from: http:// www.ibge.gov.br/home/estatistica/populacao/estimativa2012/

19. Bighetti F. Tradução e validação do Eating Attitudes Test (EAT-26) em adolescentes do sexo feminino na cidade de Ribeirão Preto. São Paulo: Universidade de São Paulo; 2003. http://dx.doi. org/10.11606/d.22.2003.tde-12042004-234230

20. Cordás TA, Castilho S. Imagem corporal nos transtornos alimentares - Instrumento de Avaliação: "Body Shape Questionnaire". Psiquiatr Biol. 1994;2(1):17-21 
21. CDC. About BMI for Adults [Internet]. 2015 [cited 2016]. Available from: http://www.cdc.gov/healthyweight/assessing/bmi/adult_bmi/ index.html

22. Garner DM, Olmsted MP, Bohr Y, Garfinkel PE. The eating attitudes test: psychometric features and clinical correlates. Psychol Med. 1982;12(4):871-8. http://dx.doi.org/10.1017/S0033291700049163

23. Nunes MA, Bagatini LF, Abuchaim AL, Kunz A, Ramos D, Silva JA, Somenzi L, Pinheiro A. Eating disorders: considerations about the Eating Attitudes Test (EAT). Rev ABP-APAL. 1994;16(1):7-10.

24. Cooper PJ, Taylor MJ, Cooper Z, Fairbun CG. The development and validation of the body shape questionnaire. Int J Eat Disord. 1987;6(4):485-94. http://dx.doi.org/10.1002/1098108X(198707)6:4<485::AID-EAT2260060405>3.0.CO;2-O

25. Martins CR, Pelegrini A, Matheus SC, Petroski EL. Insatisfação com a imagem corporal e relação com estado nutricional, adiposidade corporal e sintomas de anorexia e bulimia em adolescentes. Rev Psiquiatr Rio Gd Sul. 2010;32(1):19-23. http://dx.doi.org/10.1590/ S0101-81082010000100004

26. Jesus G, Oliveira L, Perini A, Cardoso B, Jesus B. Avaliação da satisfação com a auto-imagem corporal e presença de transtornos alimentares entre adolescentes escolares de ambos os sexos. Ulbra Mov. 2010;1(2):23-40.

27. Latner JD, Wilson RE. Obesity and body image in adulthood. In: Cash T, Smolak L. Body image: a handbook of science, practice, and prevention. New York: Guilford; 2011.

28. Lengerke T, Mielck A, Group KS. Body weight dissatisfaction by socioeconomic status among obese, preobese and normal weight women and men: results of the cross-sectional KORA Augsburg S4 population survey. BMC Public Health. 2012;12(342):2-11.

29. Ogden J, Evans C. The problem with weighing: effects on mood, self-esteem and body image. Int J Obes Relat Metab Disord. 1996;20(3):272-7.

30. Manetta M. Validade interna, dimensionalidade e desempenho da escala BSQ "Body Shape Questionaire" em uma população de estudantes universitários. São Paulo: Universidade Federal de São Paulo; 2002.

31. Leal G. Fatores associados ao comportamento de risco para transtornos alimentares em adolescentes na cidade de São Paulo. São Paulo: Universidade de São Paulo (USP); 2013. http://dx.doi. org/10.11606/t.6.2013.tde-08042013-094507

32. Damasceno M, Schubert A, Oliveira A, Sonoo C, Vieira J, Vieira L. Associação entre comportamento alimentar, imagem corporal e esquemas de gênero do autoconceito de universitárias praticantes de atividades físicas. Rev Bras Ativ Fís Saúde. 2011;16(2): 138-43.

33. Bosi MLM, Nogueira JAD, Uchimura KY, Luiz RR, Godoy MGC. Comportamento alimentar e imagem corporal entre estudantes de medicina. Rev Bras Educ Med. 2014;38(2):243-52. http://dx.doi. org/10.1590/S0100-55022014000200011

34. Mintem GC, Horta BL, Domingues MR, Gigante DP. Body size dissatisfaction among young adults from the 1982 Pelotas birth cohort. Eur J Clin Nutr. 2015;69(1):55-61. http://dx.doi.org/10.1038/ ejcn.2014.146
35. Lewis-Smith H, Diedrichs P, Rumsey N, Harcourt D. A systematic review of interventions on body image and disordered eating outcomes among women in midlife. Int J Eat Disord. 2016;49(1): 5-18. http://dx.doi.org/10.1002/eat.22480

36. Siervo M, Montagnese C, Muscariello E, Evans E, Stephan BC, Nasti G, Papa A, lannetti E, Colantuoni A. Weight loss expectations and body dissatisfaction in young women attempting to lose weight. J Hum Nutr Diet. 2014; 27 Suppl 2:84-9. http://dx.doi.org/10.1111/ jhn.12078

37. Curi G, Júnior C. Prevalência de insatisfação da imagem corporal em mulheres eutróficas com sobrepeso e obesas frequentadores de academia de ginástica. Rev Bras Obes Nutr Emagrecimento. 2011;5(23):17-23

38. Souto S, Ferro-Bucher NSJ. Práticas indiscriminadas de dietas de emagrecimento e o desenvolvimento de transtornos alimentares. Rev Nutr. 2006;19(6):693-704. http://dx.doi.org/10.1590/S141552732006000600006

39. Gravener JA, Haedt AA, Heatherton T, Keel P. Gender and age differences in associations between peer dieting and drive for thinness. Int J Eat Disord. 2008;41(1):57-63. http://dx.doi. org/10.1002/eat.20438.

40. Jackson K, Janssen I, Appelhans B, Kazlauskaite R, Karavolos K, Dugan S, Avery EA, Shipp-Johnson KJ, Powell LH, Kravitz HM. Body image satisfaction and depression in midlife women: the Study of Women's Health Across the Nation (SWAN). Arch Womens Ment Health. 2014;17(3):177-87. http://dx.doi.org/10.1007/s00737-0140416-9

41. Frois E, Moreira J, Stengel M. Mídias e a imagem corporal na adolescência: o corpo em discussão. Psicol Estud. 2011;16:71-7.

42. Calado M, Lameiras M, Sepulveda AR, Rodriguez Y, Carrera MV. The mass media exposure and disordered eating behaviours in Spanish secondary students. Eur Eat Disord Rev. 2010; 18(5):417-27. http:// dx.doi.org/10.1002/erv.1024

43. Andrés A, Saldaña C. Body dissatisfaction and dietary restraint influence binge eating behavior. Nutr Res. 2014;34(11):944-50. http://dx.doi.org/10.1016/j.nutres.2014.09.003

44. Coker E, Abraham S. Body weight dissatisfaction: a comparison of women with and without eating disorders. Eat Behav. 2014;15(3):453-9. http://dx.doi.org/10.1016/j.eatbeh.2014.06.014

45. Mintem GC, Gigante DP, Horta BL. Change in body weight and body image in young adults: a longitudinal study. BMC Public Health. 2015;15:222. http://dx.doi.org/10.1186/s12889-015-1579-7

46. Grogan S. Body image. Understanding body dissatisfaction in men, women and children. $2^{\underline{a}}$ ed. London: Routledge; 2008.

47. Jeffery RW, Drewnowski A, Epstein LH, Stunkard AJ, Wilson GT, Wing RR, Hill DR. Long-term maintenance of weight loss: current status. Health Psychol. 2000;19(1 Suppl):5-16. http://dx.doi. org/10.1037/0278-6133.19.Suppl1.5

48. Mond JM, Berg P, Boutelle K, Neumark-Sztainer D, Hannan PJ. Obesity, body dissatisfaction, and psycho-social functioning in early and late adolescence: findings from the Project EAT Study. J Adolesc Health. 2011;48:373-8. http://dx.doi.org/10.1016/j. jadohealth.2010.07.022 